\title{
Encoding strategy and not visual working memory capacity correlates with intelligence
}

\author{
Rhodri Cusack, Manja Lehmann, Michele Veldsman, and Daniel J. Mitchell \\ Medical Research Council Cognition and Brain Sciences Unit, Cambridge, England
}

\begin{abstract}
There is conflicting evidence on whether the capacity of visual working memory (VWM) reflects a central capacity limit that also influences intelligence. We propose that encoding strategy and, more specifically, attentional selection, underlie the correlation of some VWM tasks and IQ, and not variations in VWM itself. In Experiment 1, change detection measures of VWM were found to be contaminated by some cognitive process that depressed performance at higher set sizes, so that fewer items were remembered when eight rather than just four were presented. Measuring VWM using whole report instead gave a less variable estimate that was higher, particularly for larger set sizes. Nonverbal IQ did not correlate with this estimate of VWM capacity, but instead with the additional factor that contaminates change detection estimates. We propose that this phenomenon reflects a lack of selection during encoding. In Experiment 2, we investigated the role of rehearsal using articulatory suppression and showed that this could not account for the key differences between the procedures.
\end{abstract}

There is intense debate about the relationship between visual working memory (VWM) and intelligence, with conflicting evidence on whether or not VWM capacity reflects a central limit that is a bottleneck in higher order cognition. A procedure commonly used to measure VWM is change detection (CD; Luck \& Vogel, 1997; Phillips, 1974). In a typical experiment, a memorandum comprising a number of colored squares is presented briefly, in order to minimize chunking or the piecemeal transfer of items into phonological working memory. This is followed by a delay period that is sufficiently long (around $1 \mathrm{sec}$ ) to ensure the decay of sensory representations responsible for iconic memory, and then by a probe display. The participant must determine whether the items in the probe have the same color and position as those in the sample. When measured in this way, VWM has been found to predict fluid intelligence and educational achievement in adults and children (Cowan et al., 2005), as well as top-down control assessed using electroencephalography (EEG) (Vogel, McCollough, \& Machizawa, 2005), suggesting that it taps into a central capacity limit.

In contrast with this view is evidence from other studies that the capacity of VWM and measures of top-down control vary independently across individuals. Like in the classic studies by Sperling (1960), both Finke et al. (2005) and Peers et al. (2005) used a whole report (WR) paradigm to measure VWM. The memoranda were a set of letters presented briefly. Participants were asked to orally report all of the letters they could remember. This measure of VWM was compared with a measure of the efficiency of top-down control, which was derived from the ability to report target letters from among distractors of a different color (see Bundesen, 1990). It was concluded that VWM was independent of the efficiency of top-down control, across both a group of healthy controls (Finke et al., 2005) and a group of patients with parietal and frontal damage (Peers et al., 2005).

What might be the cause of these discrepant results? We focus on two possible explanations. The first is the different memoranda used in the VWM tasks: color and position (Cowan et al., 2005; Vogel et al., 2005) versus letters (Finke et al., 2005; Peers et al., 2005). A second is the different experimental procedures used: CD (Cowan et al., 2005; Vogel et al., 2005) versus WR (Finke et al., 2005; Peers et al., 2005).

\section{EXPERIMENT 1}

\section{Method}

The influence of procedure and memorandum type on estimates of VWM, and their relationship to intelligence and top-down control, were investigated. Forty-eight participants were tested (16 male, from the ages of 19 to 63; mean age 47). Approval for the experiment was given by the Cambridge Psychological Research Ethics Committee.

VWM was measured with a single WR and two CD procedures that were closely matched. All tasks required memory for letter identity, and one additionally required memory for spatial location. Identical memoranda displays were presented, with set sizes of

R. Cusack, rhodri.cusack@mrc-cbu.cam.ac.uk 


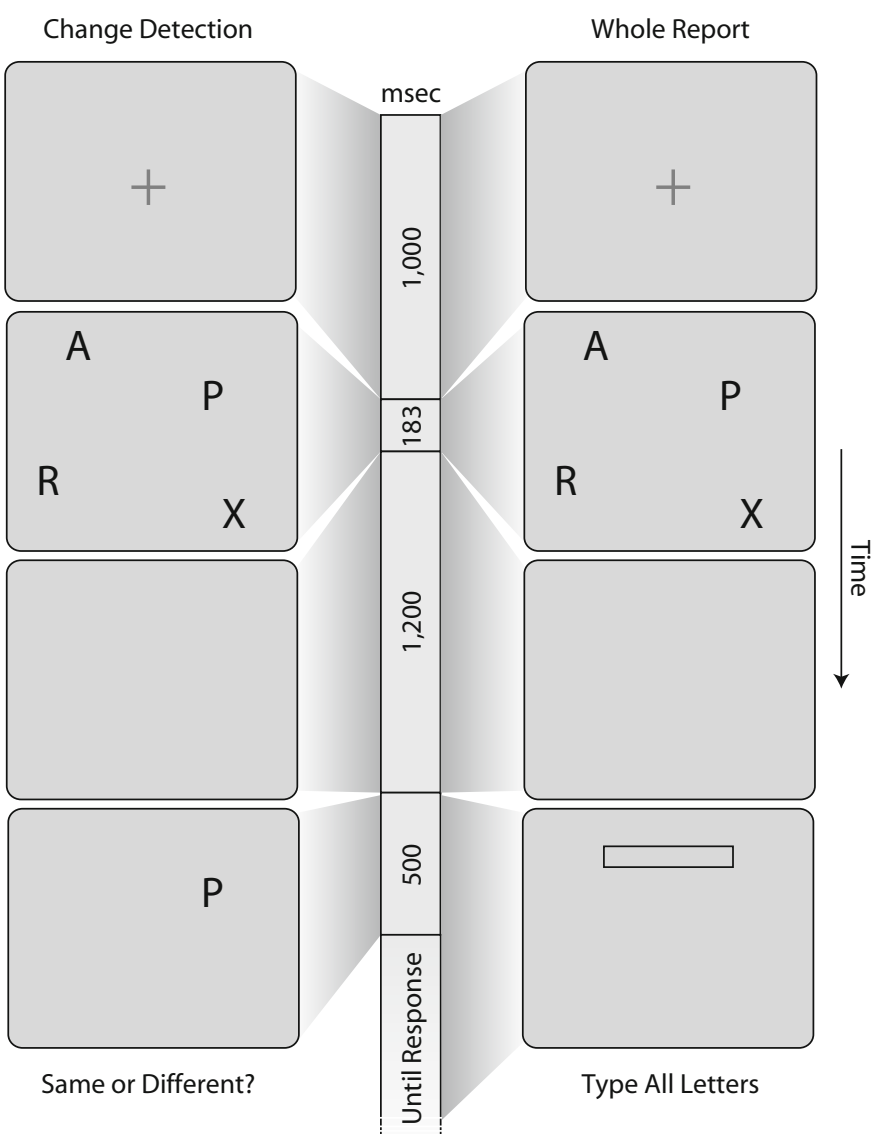

Figure 1. The structure and timing of a trial for the change detection (left) and whole report (right) tasks.

2, 3, 4, 6, or 8 letters, followed by a maintenance period (Figure 1). In the WR task for letters (VWM-WR-L), after the delay period, a box appeared and participants were asked to type all of the letters they remembered, using a standard keyboard, and then to press "Enter." In one of the CD tasks (VWM-CD-L), a probe display was presented that comprised a single letter at the location of one of the letters in the memorandum display (chosen at random), and participants were asked to press one of two buttons to indicate whether the probe letter had been present anywhere in the memorandum, irrespective of location. There was a $50 \%$ chance that it had been present. A response could be made at any point after the beginning of the probe display, and the next trial did not commence until after it had been made. In the second CD task (VWM-CD-LS), again, a single probe letter appeared at the location of one of the letters in the memorandum, and participants had to indicate whether it had been present at the same location. There was a $50 \%$ chance that the letter was the same at the same location, a $25 \%$ chance that the letter was present at a different location, and a $25 \%$ chance that it was a novel letter. The button mapping was counterbalanced across participants.

The number of items remembered at each set size was estimated for each procedure and was corrected for guessing. To calculate the number of letters remembered in the CD tasks, a double-high-threshold model was used, which assumes that $K_{\mathrm{CD}}$ items were remembered perfectly and the remainder were guessed $\left[K_{\mathrm{CD}}=N(H-F)\right.$, where $N=$ number of items in set, $H=$ hit rate, and $F=$ false alarm rate (Cowan, 2001)]. To calculate the number of letters remembered in the WR trials, the number of letters correctly reported was counted; from this, an estimate of the guessing rate was subtracted. This used the conservative assumption that the participants had learned the subset of 15 letters that could appear in the stimuli (hence possibly overestimating the guessing rate and slightly underestimating capacity): $K_{\mathrm{WR}}=C-E * N /(15-N)$, where $E=$ number of letters reported that were not in the display and $C=$ correctly reported letters.

To measure top-down control, we used a partial report (PR) procedure (Bundesen, 1990; Sperling, 1960) in which the stimuli were identical to the WR with set size 6 , except that three of the letters were white and three were black. After a delay period that matched the VWM tasks, a box appeared and participants were asked to type all of the letters in a target color. For half of the participants, the target color was fixed as white, and for the other half, it was black. As a summary measure of top-down control, we used alpha-prime (Duncan et al., 1999), which gives an estimate of "distractibility" (i.e., higher scores correspond to worse top-down control). It contrasts performance for WR with three and six items with PR in which three target items had to be selected from three distractors. If selection were perfect, PR would yield the same performance as that of three-item WR; if there were no selection at all, performance per item would be the same as in the six-item WR.

The order of the four blocks - VWM-WR-L, VWM-CD-L, VWM-CD-LS, and PR - was counterbalanced across participants. Across all of these blocks, the sample displays were $183 \mathrm{msec}$; the screen background was midgray; letters were randomly chosen from the set ABDEFGHJKMNQRTY; and their color (black or white) in the VWM blocks was matched to the target color in the partial report. Letters were presented in uppercase Arial font with the height $\sim 3.8^{\circ}$ visual angle at a location randomized within a rectangle $\sim 28^{\circ} \times 37^{\circ}$ visual angle centered at the middle of the screen, subject to the con- 


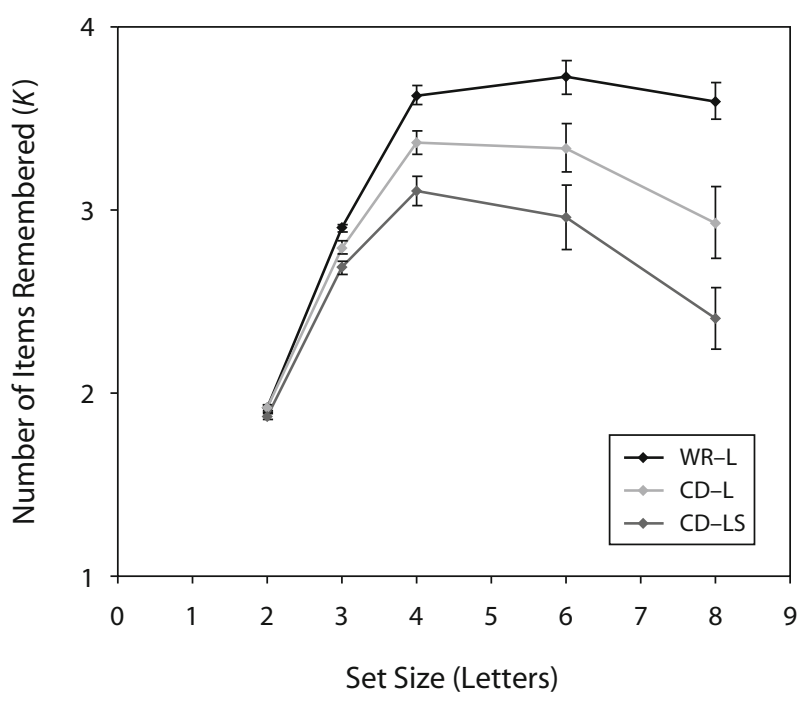

Figure 2. The number of items remembered as a function of set size for three different VWM tasks. WR-L, whole report for letter identity; CD-L, change detection for letter identity; CD-LS, change detection for letter identity and spatial position.

straint that the centers of two letters could not be closer than $\sim 6.7^{\circ}$. Since report takes longer than making a same-different response, there were fewer trials per block for WR and PR (10 practice + 120 main block; total duration [including instruction period] around $18 \mathrm{~min}, 40 \mathrm{sec})$ than there were for $\mathrm{CD}(20+240 ; 19 \mathrm{~min}, 30 \mathrm{sec})$.

Nonverbal intelligence was estimated using Cattell's (1973) culture fair.

\section{Results}

As can be seen in Figure 2, there were considerable differences in estimates from the three VWM tasks. A task (3 levels) $\times$ set size (5 levels) ANOVA on $K$ confirmed effects of task $[F(2,94)=35.4, p<.001]$ and of set size $[F(4,188)=103.5, p<.001]$, along with an interaction $[F(8,376)=8.39, p<.001]$.

Strikingly, there was a clear difference between the VWM-WR-L and VWM-CD-L conditions, which required memory for identical information, but assessed it in different ways. Three key findings were salient.

(1) WR-L gave a higher estimate than did CD-L. This pattern, seen in Figure 2, does not provide evidence for output interference in the WR procedure. Instead, there appears to be some kind of interference that spe- cifically affects CD. An ANOVA with the factors of task (VWM-WR-L and VWM-CD-L) and set size confirmed an effect of task $[F(1,47)=23.1, p<.001]$ and an interaction $[F(4,188)=6.48, p<.002]$. This difference was driven by worse performance in $\mathrm{CD}$, particularly at higher set sizes (Table 1, column a).

(2) WR-L was less variable. Although the block was faster to acquire, WR gave smaller variance (Table 1, column b).

(3) A decrease in the number of items remembered at higher set sizes was observed for CD, but not for WR. The set size dependence in CD cannot be an effect of a capacity limit alone, and instead suggests that the measure is contaminated by some additional interfering cognitive process. At lower set sizes, as more items are presented, more are remembered, for all procedures. But, above four items, although the WR condition stays approximately constant at higher set sizes (consistent with a full VWM buffer), in the CD conditions, there is a decrease in the number of items remembered with increasing set size [4 to $8: \mathrm{WR}$, $t(47)=0.47$, n.s.; CD-L, $t(47)=2.4, p<.05$; CD-LS, $t(47)=5.0, p<.001]$. This decline is significantly larger for CD than for WR [task $\times$ set size (4 or 8) interaction: $\mathrm{CD}-\mathrm{L}, F(1,47)=5.0, p<.05 ; \mathrm{CD}-\mathrm{LS}, F(1,47)=21.4$, $p<.001]$.

These features suggest that the VWM-WR provides a more canonical measure of visual working memory capacity.

To understand how intelligence affects VWM, we conducted a median split by Cattell's (1973) culture fair score (mean, 34; range, 25-42). Performance in the three VWM procedures for the participants with lower (mean 31 ) and higher (mean 38) scores is shown in Figure 3. The first striking feature is that in the VWM-WR condition, there was no effect of intelligence, suggesting no fundamental relationship between VWM and aptitude [main effect of IQ, $F(1,46)=0.00$, n.s.; set $\times$ IQ interaction, $F(1,46)=0.45$, n.s.]. Second, and importantly, there was an effect of intelligence in both of the CD conditions, but this was selective for the higher set sizes [WR vs. CD-L: task $\times$ set $\times$ IQ interaction, $F(4,184)=3.1$, $p<.05$; task $\times$ IQ interaction, $F(1,46)=3.8, p=.06$; WR vs. CD-LS: task $\times$ set $\times$ IQ interaction, $F(4,184)=$ 1.66 , n.s.; task $\times$ IQ interaction, $F(1,46)=3.0, p=.09$ ]. Using IQ as a continuous measure rather than performing a median split gave a similar result [WR vs. CD-L ANCOVA: task $\times$ IQ interaction, $F(1,46)=7.41, p<.01$,

Table 1

Statistics for Experiments 1 and 2 for Individual Set Sizes

\begin{tabular}{|c|c|c|c|c|c|c|}
\hline \multirow[b]{2}{*}{$\begin{array}{c}\text { Set } \\
\text { Size }\end{array}$} & \multicolumn{5}{|c|}{ Experiment 1} & \multirow[b]{2}{*}{$\begin{array}{c}\text { Experiment } 2 \\
\text { (f) } \\
\text { Correlation } \\
\text { IQ and WR-AS } \\
\text { [Spearman's } \rho(13)]\end{array}$} \\
\hline & $\begin{array}{c}\text { (a) } \\
\text { Mean } \\
\text { WR-L vs. CD-L } \\
{[t(47)]}\end{array}$ & $\begin{array}{c}\text { (b) } \\
\text { Variability } \\
\text { WR-L vs. CD-L } \\
{[F(47,47)]}\end{array}$ & $\begin{array}{c}(\mathrm{c}) \\
\text { Correlation } \\
\text { IQ and WR } \\
{[\text { Spearman's } \rho(48)]}\end{array}$ & $\begin{array}{c}(\mathrm{d}) \\
\text { Correlation } \\
\text { IQ and CD-L } \\
{[\text { Spearman's } \rho(48)]}\end{array}$ & $\begin{array}{c}(\mathrm{e}) \\
\text { Correlation } \\
\text { IQ and CD-LS } \\
{[\text { Spearman's } \rho(48)]}\end{array}$ & \\
\hline 2 & -0.11 & 0.53 & .01 & .17 & .08 & -.31 \\
\hline 3 & $3.0^{* *}$ & $3.7^{* * *}$ & .01 & -.09 & -.07 & -.30 \\
\hline 4 & $3.9^{* * *}$ & $1.6^{*}$ & -.12 & -.43 & .06 & -.54 \\
\hline 6 & $2.8^{* *}$ & $2.1^{* *}$ & -.03 & $.30^{*}$ & $.29^{*}$ & -.21 \\
\hline 8 & $3.6^{* * *}$ & $3.8^{* * *}$ & .00 & $.27(p=.07)$ & .19 & -.26 \\
\hline
\end{tabular}



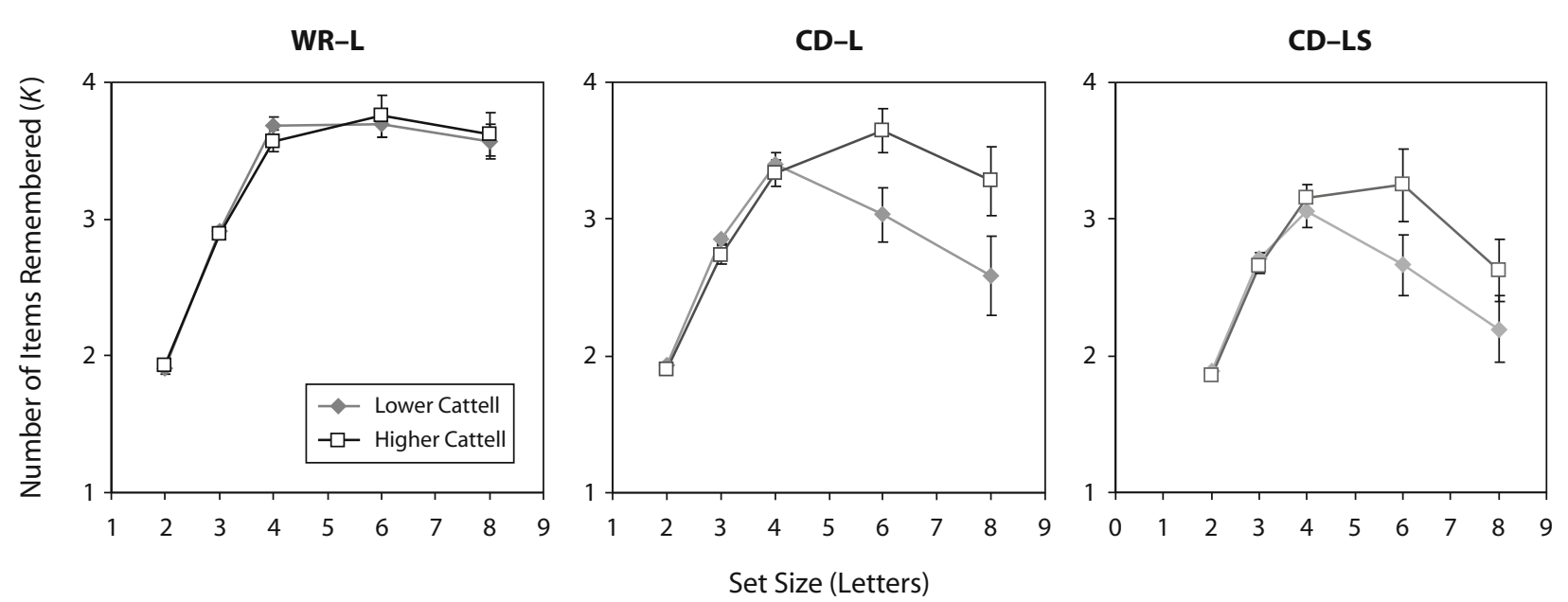

Figure 3. Performance in Experiment 1 on three VWM tasks for lower- (light gray, diamonds) and higher- (dark gray, squares) intelligence groups created by a median split on a nonverbal IQ measure, Cattell's (1973) culture fair. WR-L, whole report for letter identity; CD-L, change detection for letter identity; CD-LS, change detection for letter identity and spatial position.

Table 1, columns $\mathrm{c}-\mathrm{e}]$. This result suggests that it is the additional cognitive process that depresses performance at higher set sizes in $\mathrm{CD}$ that couples with intelligence, and not VWM per se.

A supplementary analysis was performed to investigate whether the lack of a relationship between WR and IQ might be because the WR measure is, in general, less sensitive to individual differences. As was reported above, WR is less variable than $\mathrm{CD}$, and across participants it has a smaller range (mean across set size: WR, 2.32-3.81; CD-L, 1.71-3.78; CD-LS, 1.51-3.55). The critical issue is whether this extra variance in CD reflects increased signal, or merely increased noise. In order to test for this, we used factor analysis to retrieve a single factor from the three measures (presumably underlying memory capacity), using maximum likelihood estimation on the mean across set sizes. This single factor explained a substantial proportion of the variance (67.2\%). Importantly, the factor loading for WR $(0.85)$ was higher than that for CD-L (0.60) or CD-LS (0.70). This result indicates that WR is, if anything, more sensitive than CD to underlying individual differences, and is more tightly related to each of the $\mathrm{CD}$ measures than they are to each other (this was also confirmed with the following correlations: WR with CD-L, .51; WR with CD-LS, .59; CD-L with CD-LS, .42). Similar results were obtained if IQ was partialled out prior to analysis (factor analysis, 67.2\%; weights: WR, .92; CD-L, .58; CD-LS, .65; partial correlations of WR with CD-L, .53; WR with CD-LS, .60; CD-L with CD-LS, .38). WR is at least as sensitive as $C D$ to individual differences, and the absence of a relationship between IQ and WR cannot be attributed to lower sensitivity.

Since the measure of selection, $\alpha^{\prime}$, includes a term from VWM-WR, an unbiased assessment of individual differences in VWM (as measured this way) and top-down selection could not be calculated. There were no significant correlations between $\alpha^{\prime}$ and VWM-CD-L or VWM-
CD-LS at any set size. It was also possible to examine what individual differences were associated with an ability to remember spatial information. As might be expected, there was a cost in having to also remember spatial position [CD-LS vs. CD-L, $F(1,47)=12.4, p<.001]$. To best estimate VWM capacity with $\mathrm{CD}$ while ameliorating the influence of the drop at high set sizes, for each person the maximum across set size in the number of items remembered was calculated. These did not correlate with IQ or $\alpha^{\prime}$, but the cost of also remembering spatial information $[\max (\mathrm{CD}-\mathrm{L})-\max (\mathrm{CD}-\mathrm{LS})]$ did correlate with selection $\alpha^{\prime}[\rho(48)=0.30, p<.05$; partial correlation with IQ removed, $r(45)=.30, p<.05]$, suggesting that selection and remembering spatial position share something over and above a dependency on IQ.

\section{Discussion}

WR gave a higher and less variable estimate of VWM than did CD. At high set sizes, an intriguing effect of IQ on performance was found in $\mathrm{CD}$, with participants who had lower Cattell scores remembering fewer items as more were presented beyond capacity. This deficit was absent in WR despite its requiring exactly the same memory load. What might be the cause of this depression in CD? The sensitivity of the effect to the number of items presented - even when above the capacity limit—suggests that it cannot be due to something that happens at the time of maintenance or comparison (e.g., the requirement to maintain the sample in the presence of the probe) since, by the time these phases arrive, items above capacity should already have fallen out of VWM. Instead, we propose that the critical difference is at the time of encoding: $C D$ encourages a strategy of attempting to hold a visual snapshot of the entire display, but in WR, the covert naming - or the ability to control which items are recalled on any trialencourages more selective encoding. The fact that fewer items were remembered when eight rather than four were presented in $\mathrm{CD}$ (particularly for those lower in aptitude) 
is consistent with a maladaptive lack of selection. It has been shown previously that selection is less effective in those that perform worse on VWM tasks (Cowan \& Morey, 2006; Vogel et al., 2005). We propose that this maladaptive encoding strategy contaminates the $\mathrm{CD}$ measure at higher set sizes, and that it is this - not VWM capacitythat correlates with aptitude.

\section{EXPERIMENT 2}

In Experiment 1, we found that WR encourages better selection than does $\mathrm{CD}$. This might be because during the maintenance period of WR, items were shuffled from VWM to the phonological loop, and the imminence of covert naming encouraged selection at encoding. Alternatively, in WR, participants can control which items they report, and this might have encouraged more selection than in $\mathrm{CD}$, in which random items are probed. It was the aim of Experiment 2 to contrast these two accounts by examining the effect of suppressing verbalization.

\section{Method}

Memory capacity was assessed again, using WR for letters, but with the addition of articulatory suppression, in which overt rehearsal of an interfering stimulus was used to prevent covert or overt rehearsal of the memoranda. Articulatory suppression is robust to the exact items being uttered (Baddeley, 1997, pp. 7980). Participants have been asked to rehearse a single word (e.g., "the-the-the ..."; Baddeley, 1997), numbers (two digits, overtlyVogel, Woodman, \& Luck, 2006; covertly—Todd \& Marois, 2004), or a list of items (overlearned series, "a-b-c-d"; Woodman \& Luck, 2004). To ensure fully effective suppression, Experiment 2 used a hard task, in which five spoken random digits were rapidly presented (stimulus onset asynchrony, $300 \mathrm{msec}$ ). Participants were asked to overtly rehearse them at this rate for the remainder of the trial. The digits were windowed to be $250 \mathrm{msec}$ in duration, with linear onset and offset ramps to prevent spectral splatter, and to be presented in free field at a comfortable listening level. Between the end of the last digit and the sample display, there was a delay of $1,550 \mathrm{msec}$ to allow participants to enact rehearsal. Compliance was ensured by recording participants' voices using a portable digital recorder.

It was not clear, a priori, the degree to which phonological rehearsal would affect performance on trials with a short encoding display $(183 \mathrm{msec})$; thus, to ensure an assay of the effect of articulatory suppression, trials with a longer sample display were also included $(1,000 \mathrm{msec})$. However, this condition overestimates VWM, since it allows time for visual chunking and the piecemeal transfer of items from VWM to phonological memory (Cowan, 2001).

There were 14 participants ( 7 female). All were informed prior to initial consent that their voices would be recorded during the experiment. Their mean age was 49, and their mean Cattell score was 38 . There were two blocks, one with articulatory suppression (WR-AS) and one without, with the order counterbalanced across participants. A factorial design of sample display duration (183 and 1,000 msec) and set size $(2,3,4,6,8)$ yielded 10 conditions each of 24 trials/ block, intermixed in random order. Apart from the addition of the articulatory suppression task and the modification of encoding duration in some trials, the paradigm and analysis were the same as those in Experiment 1.

\section{Results}

Figure 4 shows means and standard errors across participants. An ANOVA found main effects of set size $[F(4,52)=$

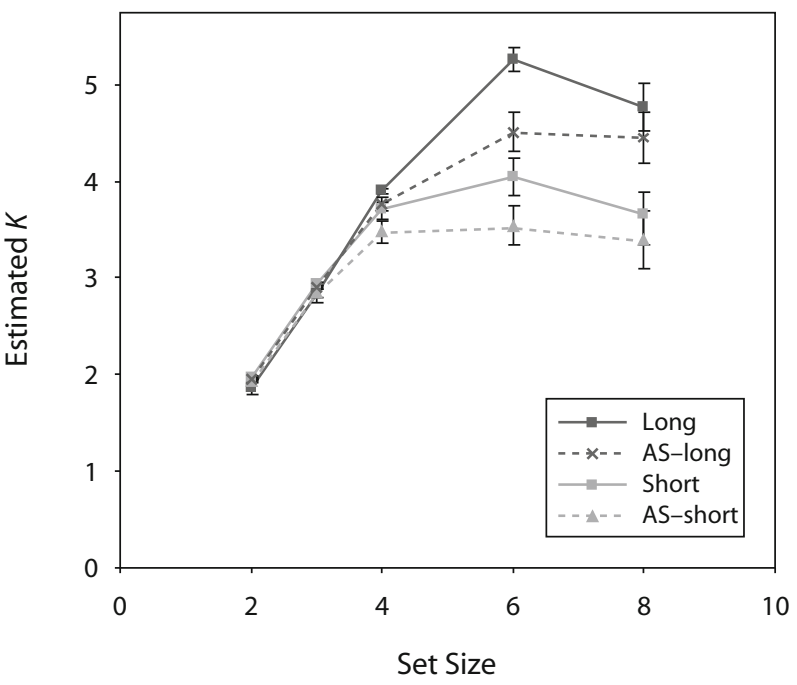

Figure 4. Estimated memory capacity as a function of set size in Experiment 2 for a whole report task with either long (1,000msec) or short (183-msec) encoding durations, and with or without articulatory suppression (AS).

79.7, $p<.001]$ and encoding duration $[F(1,13)=90.6$, $p<.001]$. The articulatory suppression task reduced performance $[F(1,13)=10.2, p<.01]$ but had no interaction with encoding duration $[F(1,13)=0.05$, n.s. $]$.

For comparability with Experiment 1, subsequent analyses then focused on the short encoding condition. Importantly, even in the presence of articulatory suppression, there was no suggestion of a downturn at high set sizes [WR-AS, short encoding, set size 4 vs. set size 8, pairedsample $t(13)=0.37$, n.s.]. This drop-off was statistically smaller than that for the group with lower Cattell scores on $\mathrm{CD}-\mathrm{L}[t(36)=2.08, p<.05]$ and similar to that for the group with higher Cattell scores $[t(36)=-0.7$, n.s. $]$. These results show that rehearsal cannot account for the absence of a drop-off at higher set sizes in WR.

A prediction from Cowan et al. (2005) is that in the presence of articulatory suppression, WR will yield a purer estimate of capacity, and so reveal a positive correlation with intelligence. There was no evidence of such a relationship, although limited conclusions should be drawn from this, because of the sample size [one-tailed test of Spearman's correlation between mean $K$ and IQ, $\rho(13)=-0.28$, n.s.; Table 1, column f].

Overall performance in the presence of articulatory suppression (WR-AS, short encoding, mean $K=3.04 \pm 0.12$ ) was reduced to the same level as CDL performance in the group with higher Cattell scores from Experiment 1 [mean $K=2.98 \pm 0.09, t(36)=0.40$, n.s.]. However, as expected, it was better than it was in the group with lower Cattell Scores for the CDL condition [mean $K=$ $2.76 \pm 0.09, t(36)=1.82, p<.05$, one-tailed]

\section{Discussion}

There was no suggestion that articulatory suppression had an impact on set sizes beyond capacity more than on 
those around capacity. This result suggests that rehearsal of the memoranda is not required to encourage the selective strategy seen in WR. Instead, we propose that the key characteristic is that the paradigm allows participants to choose which items they report, in contrast with the random probing of CD.

Performance of a hard articulatory suppression task reduced performance in WR. This reduction might be a general dual-task decrement. Rehearsing digits has been shown before to interfere with general reasoning (Baddeley, 1997) and may have an impact on executive control to the detriment of many aspects of the memory task. Alternatively, there might have been a more specific impact of articulatory suppression on maintenance. The bottleneck in WR for letters is primarily VWM (e.g., Bundesen, 1990; Cowan, 2001; Sperling, 1960), but phonological rehearsal may contribute to maintenance for the remainder of the memory delay, in which an occasional item may drop from VWM. The articulatory suppression task will have prevented such rehearsal.

\section{GENERAL DISCUSSION}

The WR procedure gives a less variable and more easily interpretable estimate of VWM capacity than does the $\mathrm{CD}$ procedure. $\mathrm{CD}$ appears to be contaminated by some additional cognitive process that depresses performance at higher set sizes. The magnitude of this contamination was found to be greater in people who performed worse on an intelligence test, but there was no relationship between VWM and intelligence per se. Experiment 2 showed that rehearsal cannot account for the absence of a drop-off at higher set sizes in WR.

In Experiment 1, the overall performance in WR was better than that for CD. In Experiment 2, the presence of a difficult articulatory suppression task reduced overall performance on WR to a level similar to that for CD in Experiment 1. It is not clear whether this reduction is because of general dual-task interference or, more specifically, because of suppression of rehearsal.

The inference that there is a difference in encoding strategy between CD and WR (see the Discussion section of Experiment 1) was confirmed in a subsequent experiment from our laboratory that has not yet been published. In this experiment, $\mathrm{CD}-\mathrm{L}$ and $\mathrm{WR}-\mathrm{L}$ trials were randomly intermixed so that participants did not know until they were probed how they would have to respond. Doing this led to a marked reduction in the difference between the procedures. This reduction suggests that worse $\mathrm{CD}$ in participants with a lower Cattell score is more likely due to the adoption of a suboptimal strategy of trying to encode everything, rather than the result of a fundamental inability to select appropriately.

Studies using verbal memoranda have found that complex measures combining storage and processing correlate more strongly with intelligence than do simple measures of storage alone (Baddeley, 1997). Both Miyake, Friedman, Rettinger, Shah, and Hegarty (2001) and Cowan et al. (2005) investigated whether the same is true for visuospatial memory, and found that it was not, since both simple and more complex visuospatial measures correlated with intelligence to the same degree. They concluded that there is an asymmetry between verbal and visuospatial storage, with the latter being fundamentally dependent on central processes related to intelligence. Our findings suggest a possible reinterpretation: Perhaps even in simple tasks intelligence had an effect on strategic choices, rather than being related to VWM per se. Interestingly, Miyake et al.'s tasks focused more on spatial memory, and our findings may have implications for this related field of study.

Given the parsimonious explanation of maladaptive selection, a surprising feature of the existing data set is that our measure of top-down selection in Experiment 1 did not correlate with performance in the $\mathrm{CD}$ procedures. It might be that, as was discussed previously, the effect of intelligence on CD is mediated by differences in strategy, rather than in the ability to select per se, and that when the task instructions explicitly require selection, the relationship is removed. This is consistent with the findings of both Gold et al. (2006) and Cowan, Morey, AuBuchon, Zwilling, and Gilchrist (in press). They found a trend of drop-off in CD at high set sizes, but good performance on an explicit selection task. The partial report selection measure did show associations in other respects: Better $\alpha^{\prime}$ was correlated with less of a decline in performance when space also had to be remembered in a CD task. This result might be because both measures tap into spatial representations, and it may have further contributed to the association between selection and VWM measures observed in tasks that required memory for spatial position (see, e.g., Vogel et al., 2005).

Many CD experiments in the literature neither require that participants use the position information (as in CDLS) nor require that they ignore irrelevant changes in position (as in CD-L). However, a drop in CD at high set sizes has been observed in experiments where a single probe is always presented at fixation (e.g., Chee \& Chuah, 2007), where a single item is probed but its position is always kept similar to that of the sample (see, e.g., Song \& Jiang, 2006), or when all of the items are probed and their positions kept constant (e.g., Gold et al., 2006).

Although the present report has focused on the important differences between procedures, it should be noted that there were significant commonalities across the measurements, as was evident from the good correlations and strong single-factor model. Reassuringly, both procedures reflect VWM to a substantial degree.

In summary, the results of the present study suggest that VWM capacity in itself is not related to cognitive aptitude, but that CD may tap into maladaptive encoding strategies that have an impact on performance at higher set sizes.

\section{AUTHOR NOTE}

We thank Alejandro Vicente-Grabovetsky for assistance with computer programming, John Duncan and Daniel Bor for insightful comments, and Ian Nimmo-Smith and Peter Watson for statistical advice. Address correspondence to R. Cusack, MRC CBU, 15 Chaucer Rd., Cambridge CB2 7EF, England (e-mail: rhodri.cusack@mrc-cbu.cam.ac.uk). 


\section{REFERENCES}

Baddeley, A. D. (1997). Human memory: Theory and practice. Hove, U.K.: Psychology Press.

Bundesen, C. (1990). A theory of visual attention. Psychological Review, 97, 523-547.

Cattell, R. B. (1973). Measuring intelligence with the culture fair tests. Champaign, IL: Institute for Personality and Ability Testing.

Chee, M. W. L., \& ChuAh, Y. M. L. (2007). Functional neuroimaging and behavioral correlates of capacity decline in visual short-term memory after sleep deprivation. Proceedings of the National Academy of Sciences, 104, 9487-9492.

CowAN, N. (2001). The magical number 4 in short-term memory: A reconsideration of mental storage capacity. Behavioral \& Brain Sciences, 24, 87-185.

Cowan, N., Elliott, E. M., Saults, J. S., Morey, C. C., Mattox, S., Hismuatullina, A., \& Conway, A. R. A. (2005). On the capacity of attention: Its estimation and its role in working memory and cognitive aptitudes. Cognitive Psychology, 51, 42-100.

COWAN, N., \& Morey, C. C. (2006). Visual working memory depends on attentional filtering. Trends in Cognitive Sciences, 10, 139-141.

Cowan, N., Morey, C. C., AuBuchon, A. M., Zwilling, C. E., \& GilCHRIST, A. L. (in press). Seven-year-olds allocate attention like adults unless working memory is overloaded. Developmental Science.

Duncan, J., Bundesen, C., Olson, A., Humphreys, G., Chavda, S., \& ShibuYA, H. (1999). Systematic analysis of deficits in visual attention. Journal of Experimental Psychology: General, 128, 450-478.

Finke, K., Bublak, P., Krummenacher, J., Kyllingsbaek, S., MülLER, H. J., \& SCHNEIDER, W. X. (2005). Usability of a theory of visual attention (TVA) for parameter-based measurement of attention I: Evidence from normal subjects. Journal of the International Neuropsychology Society, 11, 832-842.

Gold, J. M., Fuller, R. L., Robinson, B. M., McMahon, R. P., Braun, E. L., \& LUCK, S. J. (2006). Intact attentional control of working memory encoding in schizophrenia. Journal of Abnormal Psychology, 115, 658-673.

LuCK, S. J., \& VoGel, E. K. (1997). The capacity of visual working memory for features and conjunctions. Nature, 390, 279-281.

Miyake, A., Friedman, N. P., Rettinger, D. A., Shah, P., \& HegARTY, M. (2001). How are visuospatial working memory, executive functioning, and spatial abilities related? A latent-variable analysis. Journal of Experimental Psychology: General, 130, 621-640.

Peers, P. V., Ludwig, C. J., Rorden, C., Cusack, R., Bonfiglioli, C., Bundesen, C., ET AL. (2005). Attentional functions of parietal and frontal cortex. Cerebral Cortex, 15, 1469-1484.

Phillips, W. A. (1974). On the distinction between sensory storage and short-term visual memory. Perception \& Psychophysics, 16, 283-290.

Song, J.-H., \& JiAng, Y. V. (2006). Motion tracking modulates capacity allocation of visual working memory. Psychonomic Bulletin \& Review, 13, 1011-1015.

SPERLING, G. (1960). The information available after brief visual presentation. Psychological Monographs, 74, 1-29.

TodD, J. J., \& Marois, R. (2004). Capacity limit of visual short-term memory in human posterior parietal cortex. Nature, 428, 751-754. doi: 10.1038 /nature 02466

Vogel, E. K., McCollough, A. W., \& Machizawa, M. G. (2005). Neural measures reveal individual differences in controlling access to working memory. Nature, 438, 500-503.

Vogel, E. K., Woodman, G. F., \& Luck, S. J. (2006). The time course of consolidation in visual working memory. Journal of Experimental Psychology: Human Perception \& Performance, 32, 1436-1451. doi:10.1037/0096-1523.32.6.1436

Woodman, G. F., \& LucK, S. J. (2004). Visual search is slowed when visuospatial working memory is occupied. Psychonomic Bulletin \& Review, 11, 269-274.

(Manuscript received May 30, 2008; revision accepted for publication February 18, 2009.) 